\title{
ESTUDO SOBRE FATORES \\ INFLUENCIADORES DA GOVERNANÇA SUPRAEMPRESARIAL DO SEBRAE E $O$ IMPACTO NOS RESULTADOS DAS EMPRESAS ENVOLVIDAS
}

Luisa Silva Vidigal ${ }^{1}$

Henrique Cordeiro Martins ${ }^{1}$

Alexandre Teixeira Dias ${ }^{1}$

${ }^{1}$ UNIVERSIDADE FUMEC 


\title{
ESTUDO SOBRE FATORES INFLUENCIADORES DA GOVERNANÇA SUPRAEMPRESARIAL DO SEBRAE E O IMPACTO NOS RESULTADOS DAS EMPRESAS ENVOLVIDAS
}

\begin{abstract}
Resumo: Mudanças nos formatos de negócios vêm contribuindo para o surgimento de novos modelos de governança empresarial com a capacidade de prover orientação estratégica e influenciar decisões de grupos de empresas. Neste contexto, o estudo teve como objetivo analisar os fatores, confiança, atuação em rede e recursos internos, influenciadores da governança supraempresarial do SEBRAE e o impacto nos resultados das empresas envolvidas. Para alcance do objetivo, realizou-se uma pesquisa descritiva, adotando um modelo hipotético, com análise quantitativa, coleta de dados e aplicação de testes. Foi utilizado um questionário padronizado por meio do método survey com a participação de 205 indivíduos, sendo os dados submetidos a tratamento estatístico por meio da Modelagem de Equações Estruturais via ferramenta PLS. No estudo, evidenciou-se que os fatores pesquisados influenciam significativamente e positivamente a governança supraempresarial, sendo capazes de explicar $73,80 \%$ da sua variabilidade e também a sua influência sobre o desempenho das empresas.
\end{abstract}

Palavras chave: Governança supraempresarial. Confiança. Rede. Recursos.

\section{Introdução}

$\mathrm{Na}$ busca por novas formas de atuação, como redes de negócios, o papel das entidades capazes de exercer uma governança supraempresarial tornou-se imprescindível na operação de grupos de empresas. A governança de entidades empresariais é formada por um sistema sólido de instituições ou corporações, com ou sem fins lucrativos, com o objetivo de gerar valor por meio ou não de instrumentos formais (MARTINS et. al., 2016; GUIMARÃES, 2009).

Zacarelli et. al., (2008) e Telles (2008) defendem a importância do papel da governança para o desempenho e para a competitividade das redes interorganizacionais, sendo o modelo da governança supraempresarial capaz de interferir no comportamento e no direcionamento estratégico das empresas abrangidas, formulando diretrizes que mantém os agrupamentos de empresas, cooperando e se beneficiando das vantagens competitivas das redes de negócios.

Neste contexto, o artigo tem como objetivo avaliar os fatores, atuação em rede, confiança e recursos internos, que influenciam a efetividade da governança supraempresarial do SEBRAE MG (Serviço Brasileiro de Apoio a Micro e Pequena Empresa de Minas Gerais), fundamentado no seu papel de orientação empresarial e de promoção do desenvolvimento dos
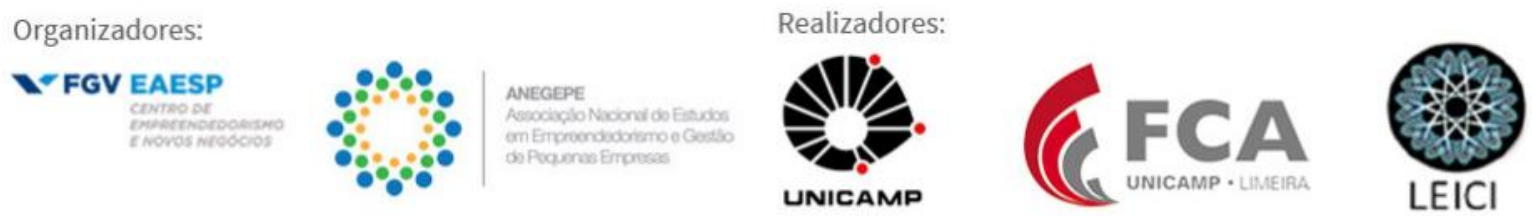
pequenos negócios, e identificar o impacto desta Instituição nos resultados das empresas envolvidas.

Para o estudo a pergunta de pesquisa que guiará a análise, visando atingir o objetivo proposto é: Quais dos fatores influenciadores da governança supraempresarial do SEBRAE MG mais impactam nos resultados das empresas abrangidas?

O modelo adotado para este artigo foi baseado no estudo realizado por Siqueira (2015) sobre o tema governança supraempresarial, que constatou como esta governança é influenciada por diferentes fatores que têm intervenção direta no desempenho dos agrupamentos, tornando-os mais ou menos competitivo.

O fator atuação em rede gera ganhos competitivos para as empresas e é um influenciador na construção de uma governança supraempresarial efetiva, enquanto a confiança aumenta as condições para a troca de informações e construção de relacionamentos a logo prazo entre as empresas e pela atuação em conjunto conseguem acessar recursos-chave para a atividade empresarial (BALESTRINI E VARGAS, 2004; GULATI et. al., 2000; MARTINS et. al., 2016)

Para a construção da fundamentação teórica, foram pesquisados conceitos relacionados às entidades supraempresariais, governança supraempresarial, desempenho, atuação em rede, confiança e recursos internos. Serão apresentados também o modelo hipotético adotado, metodologia, resultados alcançados, conclusões e contribuições.

\section{Referencial Teórico}

\subsection{Entidades Supraempresariais e Governança Supraempresarial}

A partir de uma nova perspectiva, as atuações de clusters e de redes de negócios passaram a ser identificadas como atuações supraempresariais, permitindo um conhecimento sobre a efetividade desta integração sistêmica, envolvendo atividades, gestão e estratégias independentes, sendo uma das responsabilidades das entidades supraempresariais, também nominadas redes de negócios, o estabelecimento de linhas de atuação e objetivos comuns que possibilitem cooperação entre empresas (TELLES, 2008; ZACCARELLI et. al., 2008; DUTRA, 2009; GUIMARAES et. al., 2013).

As entidades supraempresariais atuam no interesse comum das empresas com objetivo de gerar condições de competição em uma determinada rede de negócios, de maneira que se fortaleçam ao atuarem de forma integrada com as demais empresas, adquirindo novas competências e ganhos competitivos para rede e não isoladamente (GAMEIRO, 2008; ROTH et. al., 2012; DE SOUZA AGUIAR et. al., 2017).

A governança supraempresarial é um tema que vem despertando interesse de muitos pesquisadores pela forma como contribui para o desenvolvimento dos pequenos negócios que atuam em rede, considerando que a governança é um sistema que fortalece a atividade empresarial e conseqüentemente as empresas no enfrentamento de ambientes complexos (ROSSETTI; ANDRADE, 2006; GUIMARÃES, 2009).

Esta governança supraempresarial exerce influência orientadora voltado para a vitalidade do agrupamento e embora a cooperação entre as empresas proporcione benefícios adequados para todos os atores envolvidos, a escolha de um modelo de governança correto,

\section{Organizadores:}

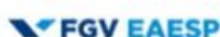

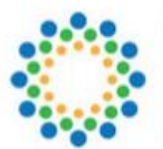

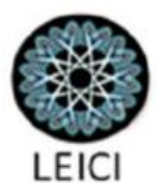


desempenha papel importante na gestão interorganizacional e no monitoramento das alianças ao longo do tempo (ALBERS, 2010; ZACARELLI et. al., 2008; GUSSONI et. al., 2015).

A forma de se organizar e de se relacionar no ambiente de negócios vem passando por mudanças e com isso surgem novos modelos de governança empresarial com abrangência sobre empresas e capaz de interferir no comportamento e nas decisões tomadas pelas empresas (DE BENEDICTO, et. al., 2008; GUIMARÃES, 2013).

Uma vez que a governança é vista como uma variável fundamental para o funcionamento e a permanência das redes interorganizacionais, é imprescindível discutir como essa governança deve ser organizada e de que forma pode impactar no desempenho dos seus membros e garantir o crescimento das empresas em um ambiente de incertezas (WEGNER et. al., 2012; DA SILVA et. al., 2016; MARTINS et. al., 2016).

Resultados de empresas representadas pela governança são maximizados quando práticas de gestão de rede permitem que os objetivos sejam alcançados e as empresas melhorem o desempenho individual (PROVAN E KENIS, 2008; ROTH et. al.; 2012).

\subsection{Atuação em Rede}

Trabalhar em rede com outras empresas tem sido a estratégia adotada por pequenos negócios e instituições na busca por competitividade e sustentação da atividade empresarial, pela perspectiva de que nenhuma organização domina individualmente as habilidades, informações e os conhecimentos fundamentais para o desenvolvimento de seus negócios. (MARTINS et. al., 2012; SILVA et. al., 2015; SOMBERGER et. al., 2017).

A permanência no ambiente de negócios deixou de ser entre empresas e passou a ser entre redes empresariais. Nesta circunstância, a cooperação e os modelos de redes são as melhores formas de reduzir riscos e manter-se freqüentemente atualizado com informação (DUTRA et. al., 2008; SILVA et. al., 2015; FREITAS et. al., 2014).

Uma rede de negócios começa a se formar quando existe uma cumplicidade nas relações de negócios entre empresas e assim conseguem obter ganhos de competitividade que dificilmente alcançariam isoladamente (BALESTRINI E VARGAS, 2004; BOAVENTURA E SIQUEIRA, 2008).

O papel da rede interorganizacional é proporcionar uma relação eficaz entre os atores de forma que, quanto mais intenso o relacionamento mais importante ele se torna para todos os envolvidos, promovendo a complementaridade de competências a favor de um de objetivo comum (HALL, 2004; BALESTRINI et. al., 2005).

\subsection{Confiança}

A confiança é considerada um elo importante no êxito organizacional e a sua ausência é considerada um fator desarticulador para qualquer rede interorganizacional, sendo um elemento essencial para interação e desenvolvimento das empresas. As transações comerciais e as ações coletivas tendem a acontecer, quando a confiança é reconhecida pelas partes envolvidas (CLARO E CLARO, 2004; HITT; IRELAND; HOSKISSON, 2003; KLEIN E PEREIRA, 2014; FILHO et. al., 2015).
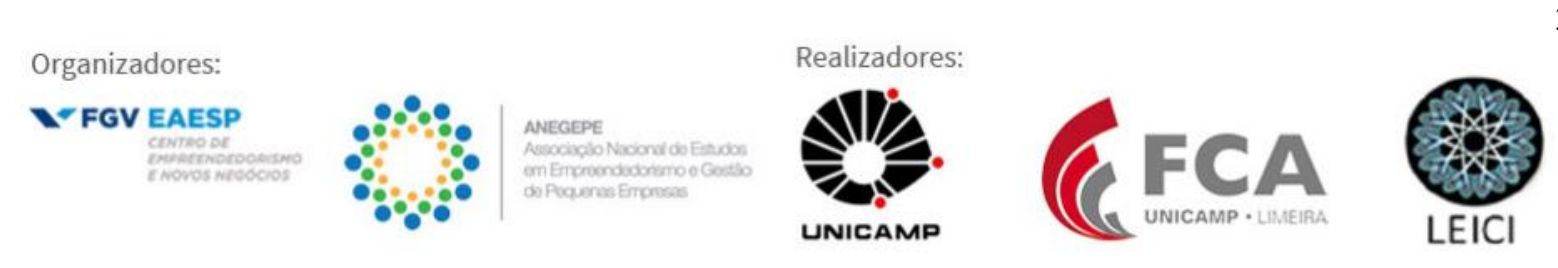
Para Child, Faulkner e Tallman (2005), a cooperação entre organizações cria uma dependência mútua entre elas, sendo a confiança elemento fundamental para se ter sucesso. $\mathrm{E}$ na certeza de que uma organização possui competência para contribuir no alcance de objetivos coletivos dos envolvidos da rede, a confiança torna a tomada de decisão mais eficiente, evitando conflitos e amenizando qualquer possibilidade de perde da cooperação (MCEVILY; PERRONE; ZAHEER, 2003; MACIEL; REINERT; CAMARGO, 2011).

As negociações são menos dispendiosas em condições de alta confiança interorganizacional (ZAHHER, A.; MCEVILY, B.; PERRONE, V, 1998).

Segundo Carnaúba (2014) e Celani et al., (2015), estudos associam alto nível de confiança com altos níveis de desempenho dos agrupamentos, sendo a confiança responsável pela construção de relacionamentos duradouros.

Diante do exposto, a confiança, como um fator influenciador de uma governança é uma variável intrínseca para os membros que fazem parte da rede. As relações são marcadas pela confiança e que pode ser entendida como a percepção que cada membro tem de que o outro não irá explorar suas vulnerabilidades (CARNAUBA, 2013).

\subsection{Recursos Internos e Desempenho}

No desenvolvimento de uma rede interorganizacional é imprescindível que os membros percebam a governança como uma forma legítima de condução da rede a partir da qual é possível gerar benefícios coletivos (WEGNER, 2011; MARIANO et. al., 2012).

As empresas ao buscarem formas de cooperação procuram melhorar seu desempenho, mas, em avaliações reais, muitas vezes não estabelecem estruturas eficientes para gerenciar a aliança com outras empresas (ZIMMER et. al., 2008; VERCECHO et. al.,2012; CUNHA E CORREA, 2013).

Improvável que uma rede perpetue ao longo do tempo se os ganhos desejados não forem obtidos e nesta circunstância, a cooperação e os modelos de redes seriam a melhor forma de reduzir riscos, dividir custos e aumentar resultados (VESCHOORE; BALESTRINI, 2010; FREITAS et. al.; 2014).

A avaliação de desempenho é parte integrante das atividades de gestão da rede e que somente podem ser melhoradas as práticas de gestão à medida que são mensuradas, sendo necessário para um processo avaliativo eficaz o conhecimento aprofundado do ambiente em que as empresas estão inseridas (WEGNER, 2011; VALMORBIDA et. al., 2011; BONATO et. al., (2017).

Os recursos das empresas só podem ser uma fonte de vantagem competitiva quando são valiosos e assim permitem que uma empresa conceba ou programe estratégias que melhorem sua eficácia, sendo o desempenho determinado pela estrutura e estratégias adotadas pela empresa (BARNEY et. al., 1991; CARNEIRO; CAVALCANTI; SILVA, 1999).

Na percepção de Balestrin, Verschoore e Perucia (2011) retornos acima da média, em determinado mercado, são resultado dos recursos (tangíveis e intangíveis) que uma empresa possui internamente.

A criação de recursos geradores de valor reside na rede de relações de uma empresa, permitindo que as empresas acessem recursos-chaves, tais como informações, capital, bens e

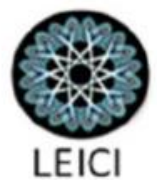


serviços de seu ambiente e possibilitando explorar oportunidades e minimizar ameaças (BARNEY, 1991; GULATI et. al., 2000).

Alguns dos principais objetivos para a interação de negócios resumem-se na melhoria das capacidades e recursos internos; levantamento de recursos e capacidades disponíveis no ambiente externo; desenvolvimento de novos recursos e habilidades por meio de alianças; aumento da efetividade do negócio e capacidade de adquirir conhecimentos (BARNEY et al., 2001; ALVES et. al., 2010; SCHIMIDT, 2013).

\section{Modelo hipotético}

Para a pesquisa, foi utilizado o modelo teórico apresentado no estudo de Siqueira (2015) com a formulação de hipóteses relacionadas aos fatores influenciadores e variáveis construindo um modelo conforme a Figura 1.

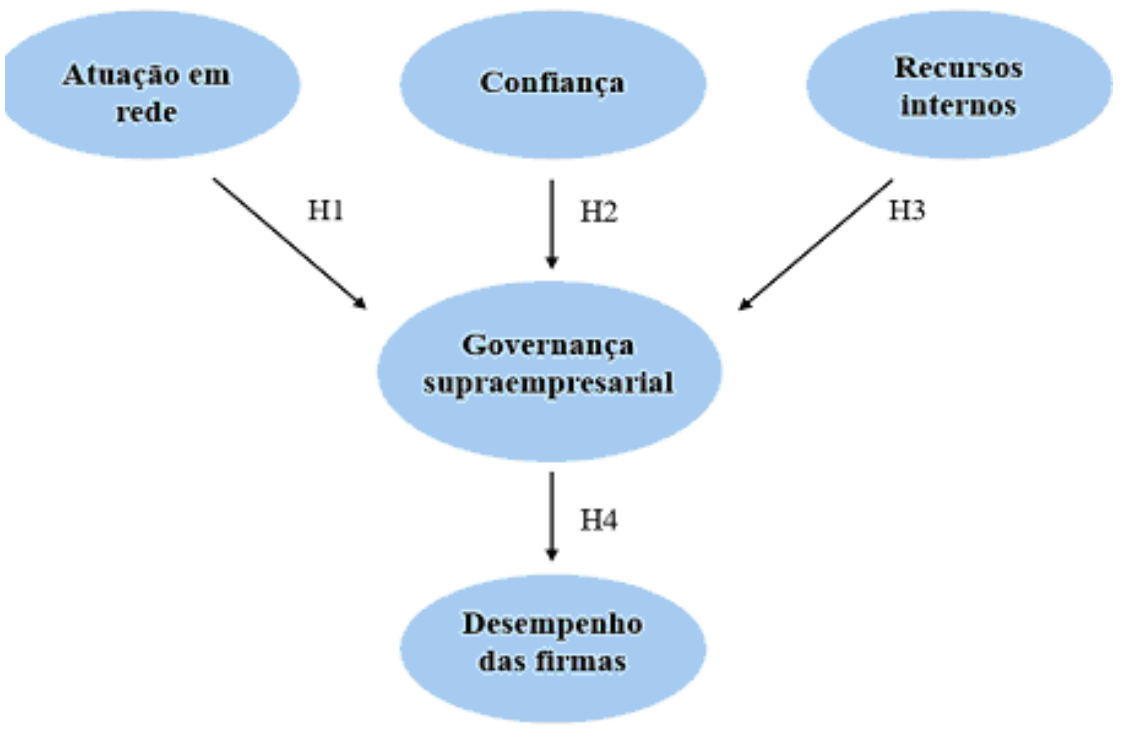

Figura 1 - Modelo teórico hipotético

Fonte: SIQUEIRA (2015), pag. 65

Hipótese 1: A atuação em rede influencia positivamente a construção de uma governança supraempresarial efetiva;

Hipótese 2: A confiança entre os entes formadores de rede tem relação positiva com a efetividade da governança supraempresarial;

Hipótese 3: Os recursos internos da entidade empresarial influenciam positivamente a efetividade da governança supraempresarial;

Hipótese 4: Uma governança supraempresarial efetiva contribui positivamente para aumentar o desempenho das empresas do agrupamento. 
Diversos autores contribuíram com conhecimento sobre o tema abordado, enfatizando a relevância dos fatores influenciadores da efetividade de uma governança supraempresarial e o seu impacto no desempenho das empresas.

Para hipótese 1 segundo Martins et. al., (2016), o constructo atuação em rede pode gerar ganhos competitivos e ser um influenciador na construção de uma governança supraempresarial efetiva.

Para a hipótese 2, o constructo confiança tem uma função importante para manutenção do agrupamento cooperativo, pois a conseqüência da quebra de confiança no relacionamento entre empresas pode resultar em medidas de controles formais, redução da confiança e encerramento da rede construída (CARANUBA, 2014).

Para a hipótese 3, conforme Hall (2004), alianças e parcerias possuem um papel fundamental na construção das vantagens competitivas baseadas em recursos da organização. É preciso investir em recursos estratégicos e desenvolver capacidades que permitam as empresas competirem em condições vantajosas. (VALLANDRO; TREZ, 2013).

Para hipótese 4, as práticas de gestão da rede permitem que os objetivos das empresas sejam alcançados melhorando o desempenho e influenciando positivamente o desempenho dos membros da rede (ROTH et. al., 2012).

$\mathrm{Na}$ construção da fundamentação teórica e contribuições, ficou evidente a existência de fatores que influenciam a governança supraempresarial e sua capacidade de gerar recursos e incentivos que contribuem no desempenho do agrupamento de empresas.

\section{Procedimento metodológico 4.1 Classificação e amostragem}

Para o estudo, foi definida a pesquisa descritiva com uma análise quantitativa, coleta de dados numéricos e aplicação de testes estatísticos. Para a coleta de dados, utilizou-se de questionário padronizado por meio do método survey. O questionário foi distribuído durante ações desenvolvidas pelo SEBRAE Minas na sua Matriz e nos Escritórios distribuídos no interior do Estado. Foi realizado o pré-teste por meio de amostragem não probabilística, em um grupo de 15 (quinze) empresas para avaliação da consistência e coerência.

A amostra foi representada por 205 respondentes da pesquisa, conforme a disponibilidade, sendo constituída por empresas de diversos setores, apoiadas e atendidas em ações do SEBRAE MG. O questionário seguiu a lógica apresentada no referencial teórico, com a coleta de dados de cada fator influenciador da governança supraempresarial, distribuídos em 28 perguntas.

\subsection{Análises dos dados}

Utilizou-se da análise multivariada para tratamento e análise de dados, considerando a principal finalidade de produzir informações para tomada de decisão. Os dados foram submetidos a tratamento estatístico por meio da Modelagem de Equações Estruturais via ferramenta PLS (Partial Least Squares - Path Modeling). O funcionamento da abordagem PLS é eficiente para tamanhos amostrais relativamente pequenos e modelos complexos.

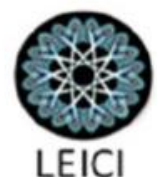


Ainda, apresenta a grande vantagem de fazer poucas suposições sobre a distribuição dos dados (CASSEL, et. al., 1999).

$\mathrm{Na}$ análise descritiva das variáveis de caracterização da amostra, foram utilizadas as frequências absolutas e relativas. Já na descrição dos itens dos constructos, foram utilizadas medidas de posição, tendência central e dispersão, sendo uma das medidas utilizadas o intervalo percentílico bootstrap com 95\% de confiança. O método bootstrap (Efron e Tibishirani, 1993) é muito utilizado na realização de inferências quando não se conhece a distribuição de probabilidade da variável de interesse.

Para avaliar a consistência interna (confiabilidade) dos constructos, foi utilizado o coeficiente Alfa de Cronbach (A.C) e a Confiabilidade Composta (C.C), que são indicadores baseado na intercorrelação dos itens do constructo. Segundo Nunally \& Bernstein (1994), valores acima de 0,70 são considerados satisfatórios e valores acima de 0,60 são aceitáveis em pesquisas exploratórias e na avaliação da qualidade do ajuste do modelo foram utilizados o $\mathrm{R}^{2}$ e o GoF (Tenenhaus, et. al., 2004).

\subsection{Instituição analisada}

O SEBRAE - Serviço Brasileiro de Apoio à Micro e Pequena Empresa é uma entidade privada que promove a competitividade e o desenvolvimento sustentável dos empreendimentos de micro e pequeno porte. Há mais de 40 anos, atua com foco no fortalecimento do empreendedorismo e na aceleração do processo de formalização da economia por meio de parcerias com os setores públicos e privados, programas de capacitação, acesso ao crédito e à inovação e estímulo ao associativismo e cooperação.

O SEBRAE tem a sua importância no ambiente produtivo e no cenário econômico, sendo um serviço de promoção de pequenos negócios com abrangência nacional, o que evidência a viabilidade de se realizar a análise de sua efetividade enquanto parte da governança supraempresarial.

Percebe-se que no ambiente empresarial, as empresas mais competitivas têm privilegiado a atuação em rede, na qual o compartilhamento de conhecimentos e informações eleva a capacidade de competir (SEBRAE, 2017).

\section{Análise descritiva dos resultados}

Pesquisa realizada com 205 indivíduos que responderam 28 questões relacionadas à 5 constructos (confiança, atuação em rede, recursos internos, governança supraempresarial e desempenho). A maioria dos indivíduos $(68,78 \%)$ da amostra coletada era sócio da empresa em que trabalhavam com a maioria $(40,98 \%)$ atuando no setor de varejo. Dos indivíduos pesquisados 52, 68\% trabalham em empresas de pequeno porte, $30,73 \%$ em microempresas, evidenciando uma amostra formada por pequenos negócios, conforme tabela 1. 
Tabela 1 - Descritiva das variáveis caracterizadoras da amostra

\begin{tabular}{llcc}
\hline \multicolumn{2}{c}{ Variáveis } & $\mathbf{N}$ & $\mathbf{\%}$ \\
\hline \multirow{4}{*}{ Cargo } & Diretor & 19 & $9,27 \%$ \\
& Gerente & 35 & $17,07 \%$ \\
& Sócio & 141 & $68,78 \%$ \\
& Outros & 10 & $4,88 \%$ \\
\hline \multirow{4}{*}{ Setor da empresa } & Agronegócios & 22 & $10,73 \%$ \\
& Indústria & 32 & $15,61 \%$ \\
& Serviços & 67 & $32,68 \%$ \\
& Varejo & 84 & $40,98 \%$ \\
\hline \multirow{4}{*}{ Tamanho da empresa } & Micro & 63 & $30,73 \%$ \\
& Pequena & 108 & $52,68 \%$ \\
& Média & 31 & $15,12 \%$ \\
& Grande & 3 & $1,46 \%$ \\
\hline \hline
\end{tabular}

Fonte: Dados da pesquisa

Em relação ao tamanho das empresas, a maioria dos entrevistados se enquadrou como micro e pequenas empresas $(83,41 \%)$, confirmando o papel da instituição SEBRAE MG no atendimento aos pequenos negócios.

Vale ressaltar que o grande número de respondentes da pesquisa de campo $(78,05 \%)$ era sócio e diretores, o que traz credibilidade na análise, uma vez que ocupam posições estratégicas, de decisões e conhecem bem a realidade dos seus negócios.

A análise dos itens dos constructos é apresentada na tabela 2. Com o intuito de apresentar e comparar os itens, foi utilizado o intervalo bootstrap com $95 \%$ de confiança. Logo, intervalos estritamente menos que 0 indicam que os indivíduos tenderam a discordar o item, enquanto intervalos estritamente maiores que 0 indicam uma tendência a concordar com o item e intervalos que contêm o 0 não indicam concordância nem discordância com o item. Logo, vale ressaltar que:

No constructo confiança, os entrevistados tenderam a concordar com todos os itens. Houve diferença significativa do item Conf.5 ("Estar ligado ao SEBRAE diminui minha percepção de vulnerabilidade no setor em que atuo") em relação aos demais itens. Uma possibilidade para esta diferença pode estar relacionada a fatores não controláveis pela empresa e pelo SEBRAE MG como políticas econômicas, variações cambiais, mercado, recursos logísticos entre outros.

Também para o fator atuação em rede e recursos internos, obteve-se a concordância da maioria dos itens o que se leva a concluir que estes fatores influenciam positivamente na efetividade da governança supraempresarial. Houve diferença significativa do item Rede 9 ("A estrutura relacional entre as empresas e o SEBRAE MG pode ser considerada um recurso de difícil imitação que aumenta a competitividade da minha empresa") em relação aos demais itens conforme tabela 2. Pressupõe-se que como existe uma relação com o SEBRAE MG, outras formas de organização existem e podem contribuir para a competitividade das empresas. 
Segundo Boaventura e Siqueira (2008), as empresas ao considerarem o ambiente competitivo, devem aproximar de outras empresas para uma atuação conjunta, com a qual há maior possibilidade de ganho em rede.

Nos fatores governança supraempresarial e desempenho, os respondentes também tenderam a concordar com todos os itens pesquisados. Em relação ao desempenho, os indivíduos tenderam a concordar menos com o item Desem.23, ("Obtenho ganhos financeiros e ou econômicos por meio da relação com o SEBRAE”) conforme a tabela 2. Uma perspectiva para que a relação com o SEBRAE MG não garanta ganhos financeiros e econômicos pode estar ligada também a fatores externos como mercado, ambiente econômico, recursos logísticos entre outros que podem afetar os resultados das empresas. Segundo Wegner (2011), os resultados obtidos pelas empresas integrantes das redes não podem ser garantidos tão somente pelos efeitos da rede. É inquestionável que existem influências externas e outras relacionadas às próprias empresas, as quais afetam seu desempenho.

Tabela 2 - Descritiva das variáveis dos constructos

\begin{tabular}{|c|c|c|c|c|}
\hline Constructos & Itens & Média & D.P. & I.C $-95 \%^{1}$ \\
\hline \multirow{5}{*}{ Confiança } & Conf.1 & 0,77 & 0,32 & {$[0,73 ; 0,82]$} \\
\hline & Conf.2 & 0,64 & 0,40 & {$[0,58 ; 0,69]$} \\
\hline & Conf.3 & 0,78 & 0,32 & {$[0,73 ; 0,83]$} \\
\hline & Conf.4 & 0,81 & 0,30 & {$[0,76 ; 0,84]$} \\
\hline & Conf.5 & 0,53 & 0,54 & {$[0,46 ; 0,61]$} \\
\hline \multirow{5}{*}{ Atuação em Rede } & Rede.6 & 0,80 & 0,28 & {$[0,76 ; 0,83]$} \\
\hline & Rede.7 & 0,71 & 0,37 & {$[0,66 ; 0,76]$} \\
\hline & Rede. 8 & 0,56 & 0,44 & {$[0,50 ; 0,62]$} \\
\hline & Rede.9 & 0,41 & 0,50 & {$[0,33 ; 0,47]$} \\
\hline & Rede.10 & 0,70 & 0,40 & {$[0,65 ; 0,76]$} \\
\hline \multirow{5}{*}{ Recursos internos } & Rec.11 & 0,77 & 0,31 & {$[0,73 ; 0,81]$} \\
\hline & Rec.12 & 0,70 & 0,32 & {$[0,65 ; 0,74]$} \\
\hline & Rec.13 & 0,68 & 0,40 & {$[0,62 ; 0,73]$} \\
\hline & Rec.14 & 0,72 & 0,34 & {$[0,67 ; 0,77]$} \\
\hline & Rec.15 & 0,60 & 0,48 & {$[0,53 ; 0,66]$} \\
\hline \multirow{7}{*}{ Governança Supraempresarial } & Govern.16 & 0,60 & 0,42 & {$[0,54 ; 0,65]$} \\
\hline & Govern.17 & 0,56 & 0,43 & {$[0,49 ; 0,61]$} \\
\hline & Govern.18 & 0,69 & 0,44 & {$[0,63 ; 0,75]$} \\
\hline & Govern.19 & 0,72 & 0,33 & {$[0,67 ; 0,77]$} \\
\hline & Govern.20 & 0,65 & 0,38 & {$[0,60 ; 0,70]$} \\
\hline & Govern.21 & 0,70 & 0,35 & {$[0,65 ; 0,75]$} \\
\hline & Govern.22 & 0,72 & 0,39 & {$[0,67 ; 0,77]$} \\
\hline \multirow{6}{*}{ Desempenho das firmas } & Desem.23 & 0,46 & 0,52 & {$[0,39 ; 0,53]$} \\
\hline & Desem.24 & 0,72 & 0,37 & {$[0,67 ; 0,77]$} \\
\hline & Desem.25 & 0,68 & 0,39 & {$[0,63 ; 0,73]$} \\
\hline & Desem.26 & 0,71 & 0,34 & {$[0,66 ; 0,75]$} \\
\hline & Desem.27 & 0,72 & 0,35 & {$[0,67 ; 0,77]$} \\
\hline & Desem.28 & 0,74 & 0,36 & {$[0,70 ; 0,79]$} \\
\hline
\end{tabular}

Fonte: Dados da pesquisa 


\subsection{Equações Estruturais e validação das Hipóteses testadas}

Para o modelo de mensuração foi avaliado a confiabilidade, dimensionalidade, validade convergente e divergente no intuito de garantir a precisão das variáveis indicadoras (itens) na formação dos constructos. Na análise, conforme tabela 3, pode destacar que:

- Todos os constructos apresentaram os índices de confiabilidade A.C. ou C.C. acima de 0,70, evidenciando assim a confiabilidade dos constructos.

- Todos os constructos foram unidimensionais segundo o critério de Kaiser.

- Todos os constructos apresentaram AVE superior a 0,50, indicando validação convergente.

- Para os constructos atuação em rede e governança supraempresarial não houve validação discriminante segundo o critério de Fornell et al. (1981), porém, de acordo com o critério das cargas fatoriais cruzadas, houve validação discriminante.

Tabela 3 - Validação do modelo de mensuração

\begin{tabular}{lccccccc}
\hline \multicolumn{1}{c}{ Constructos } & Itens & A.C. & C.C. $^{\mathbf{2}}$ & Dim. $^{\mathbf{3}}$ & $\mathbf{A V E}^{\mathbf{4}}$ & $\mathbf{V C M}^{\mathbf{5}}$ \\
\hline Confiança & 5 & 0,76 & 0,84 & 1 & 0,52 & 0,51 \\
Atuação em Rede & 5 & 0,81 & 0,87 & 1 & 0,57 & 0,58 \\
Recursos internos & 5 & 0,87 & 0,91 & 1 & 0,67 & 0,66 \\
Governança Supraempresarial & 7 & 0,89 & 0,91 & 1 & 0,60 & 0,66 \\
Desempenho das firmas & 6 & 0,91 & 0,93 & 1 & 0,68 & 0,61 \\
\hline \hline
\end{tabular}

${ }^{1}$ Alfa de Cronbach; ${ }^{2}$ Confiabilidade Composta; ${ }^{3}$ Dimensionalidade; ${ }^{4}$ Variância Extraída; ${ }^{5}$ Máximo da Variância Compartilhada.

$\mathrm{Na}$ avaliação da qualidade do ajuste do modelo foram utilizados o $\mathrm{R}^{2}$ e o GoF (Tenenhaus et. al., 2004). O $\mathrm{R}^{2}$ representa em uma escala de $0 \%$ a $100 \%$ o quanto os constructos independentes explicam os dependentes, sendo que, no geral, valores de $\mathrm{R}^{2}$ de $75 \%, 50 \%$ e $25 \%$ podem ser descritos como forte, moderado e fraco (Hair et. al., 2014). Já o GoF é uma média geométrica das AVEs dos construtos e dos $\mathrm{R}^{2}$ do modelo e também varia de $0 \%$ a $100 \%$.

A Tabela 4 apresenta os resultados do modelo de mensuração. Logo, vale ressaltar que com relação à Governança Supraempresarial:

Houve influência significativa (valor-p $=0,000)$ e positiva $(\beta=0,22[0,11 ; 0,32]) \mathrm{da}$ confiança sobre a governança supraempresarial. Sendo assim, quanto maior for a confiança, melhor será a governança supraempresarial.

Houve influência significativa (valor-p $=0,000)$ e positiva $(\beta=0,25[0,13 ; 0,37])$ da atuação em rede sobre a governança supraempresarial. Sendo assim, quanto maior for a atuação em rede, melhor será a governança supraempresarial.

Houve influência significativa (valor-p $=0,000)$ e positiva $(\beta=0,49[0,38 ; 0,60])$ dos recursos internos sobre a governança supraempresarial. Sendo assim, quanto maior forem os recursos internos, melhor será a governança supraempresarial. 
A confiança, atuação em rede e recursos internos foram capazes de explicar $73,80 \%$ da variabilidade da governança supraempresarial.

Com relação ao Desempenho: houve influência significativa (valor-p $=0,000$ ) e positiva $(\beta=0,78[0,69 ; 0,87])$ da governança supraempresarial sobre o desempenho das empresas assistidas pelo SEBRAE. Sendo assim, quanto maior for a governança supraempresarial, maior será o desempenho das empresas envolvidas.

A governança supraempresarial foi capaz de explicar $60,70 \%$ da variabilidade do desempenho das empresas assistidas pelo SEBRAE.

Vale destacar também que o modelo apresentou um GoF de 64,10\% e, além disso, os intervalos de confiança bootstrap estavam de acordo com os resultados encontrados via valor$\mathrm{p}$, evidenciando assim maior validade dos resultados apresentados.

Tabela 4 - Modelo Estrutural

\begin{tabular}{llccccc}
\hline \multicolumn{1}{c}{ Endógenas } & \multicolumn{1}{c}{ Exógenas } & B & E.P.(妵 & I.C. - 95\% & Valor-p & $\mathbf{R}^{\mathbf{2}}$ \\
\hline \multirow{2}{*}{ Governança Supraempresarial } & Confiança & 0,22 & 0,05 & {$[0,11 ; 0,32]$} & 0,000 & \\
& Atuação em Rede & 0,25 & 0,06 & {$[0,13 ; 0,37]$} & 0,000 & $73,80 \%$ \\
& Recursos internos & 0,49 & 0,06 & {$[0,38 ; 0,60]$} & 0,000 & \\
\hline Desempenho das firmas & Governança Supraempresarial & 0,78 & 0,04 & {$[0,69 ; 0,87]$} & 0,000 & $60,70 \%$ \\
\hline \hline
\end{tabular}

${ }^{1}$ Erro Padrão; ${ }^{2}$ Intervalo bootstrap; GoF $=64,10 \%$.

Fonte: Dados da pesquisa

O relacionamento de empresas por meio de redes de cooperação pode ocorrer da premissa de que nenhuma empresa domina todas as habilidades para competir no mercado isoladamente e que desta forma adotam estratégias em conjunto para fortalecimento dos seus negócios (SILVA et. al., 2015; MARTINS et. al., 2012).

Tabela 5 - Hipótese do modelo

\begin{tabular}{cll}
\hline & \multicolumn{1}{c}{ Hipóteses } & Resultado \\
\hline H1 & $\begin{array}{l}\text { A atuação em rede influência positivamente a construção de } \\
\text { uma governança supraempresarial efetiva. }\end{array}$ & Confirmada \\
\hline H2 & $\begin{array}{l}\text { A confiança entre os entes formadores de rede tem relação } \\
\text { positiva com a efetividade da governança supraempresarial. }\end{array}$ & Confirmada \\
\hline H3 & $\begin{array}{l}\text { Os recursos internos da entidade empresarial influenciam } \\
\text { positivamente a efetividade da governança supraempresarial. }\end{array}$ & Confirmada \\
\hline H4 & $\begin{array}{l}\text { Uma governança supraempresarial efetiva contribui positivamente } \\
\text { para aumentar o desempenho das empresas do agrupamento. }\end{array}$ & Confirmada \\
\hline \hline
\end{tabular}

Fonte: Dados da pesquisa 


\section{Conclusões}

$\mathrm{Na}$ expectativa de gerar conhecimento sobre o tema, o objetivo de "Analisar fatores influenciadores da governança supraempresarial do SEBRAE MG e o impacto nos resultados das empresas envolvidas" foi alcançado, contribuindo para a construção do alicerce teórico.

Considerando as análises e com base no modelo hipotético adotado, confirmou-se a associação dos fatores confiança, atuação em rede e recursos internos com a governança supraempresarial e o desempenho.

Estes fatores foram capazes de explicar $73,80 \%\left(\mathrm{R}^{2}\right)$ da variabilidade da governança supraempresarial, o que significa influência positiva sobre a governança existente. Confirmou-se também o seu impacto no desempenho das empresas envolvidas, sendo capaz de explicar $60,70 \%\left(\mathrm{R}^{2}\right)$ da variabilidade do desempenho das empresas assistidas pelo SEBRAE, evidenciando assim que diferentes modelos de organização e atuação empresarial podem contribuem para alcance de resultados coletivos.

Dentre os constructos analisados o fator recursos internos teve uma maior influência sobre a governança supraempresarial, o que demonstra o quão é importante para uma rede de cooperação a atração e associação de competências e recursos possibilitando a geração de valor, validando a $\mathrm{H} 3$, conforme a tabela 5.

Igualmente as $\mathrm{H} 1, \mathrm{H} 2$ e $\mathrm{H} 4$ se confirmaram, considerando que atuação em rede fortalece o ambiente das empresas, a confiança gera credibilidade e segurança nas relações e a governança empresarial exercida pelo SEBRAE tem impacto direto na competitividade dos pequenos negócios.

As instituições ao adotarem modelos de interação conduzem as empresas a uma relação de confiança e assim, o engajamento e o relacionamento tendem a minimizar riscos e encorajar troca de idéias entre os parceiros de negócios.

A governança supraempresarial exerce um papel capaz de orientar e influenciar as decisões estratégicas dos negócios inseridos em rede seja por meio de um modelo formal ou não, mas com objetivo de contribuir com o ambiente onde está inserido.

Ficou evidenciado no estudo, conforme os dados apresentados, a relevância da governança supraempresarial para o agrupamento de empresas. A capacidade de articular, de convergir interesses, de prover recursos e incentivos na busca por competitividade, contribui para a sua presença e fortalecimento.

Espera-se, como oportunidade, que esta pesquisa incentive outras instituições de fomento e o setor produtivo a investirem e apoiarem o desenvolvimento das redes como modelo competitivo de negócios.

Tem-se como expectativa também que subsidie a academia, professores e alunos na consolidação do tema e na geração de novos estudos, uma vez que não foi encontrado na literatura pesquisa da replicação de um modelo com foco na efetividade da governança supraempresarial. 


\section{Referências}

ALBERS, S. Configurations of alliance governance Systems. Schmalenbach Business Review, n. 62, p. 204-233, 2010.

ALVES, C. A; KASSOUF P. N; NEVES G.M. A importância estratégica dos relacionamentos de negócios em redes de empresas: uma visão baseada no RBV-Resource Based View. Revista Brasileira de Marketing, v. 9, n. 2, 2010.

BALESTRIN, A.; VARGAS, L.M. A dimensão estratégica das redes horizontais de PMEs: teorizações e evidências. Revista de Administração Contemporânea, p. 203, 2004.

BALESTRIN, A; VARGAS, L.M; FAYARD, P. Criação de conhecimento nas redes de cooperação interorganizacional. Revista de Administração de Empresas, v. 45, n. 3, 2005.

BALESTRIN, A.; VERSCHOORE, J. R.; PERUCIA, A. Visão Relacional no Campo da Estratégia: Evidências Empíricas a partir das Ações Coletivas de Redes interorganizacionais. In: V Encontro de Estudos em Estratégia. Porto Alegre, 2011

BARNEY, J. B; WRIGHT, Mike; KETCHEN JR., David. The resource-based view of the firm: Ten years after 1991. Journal of management, v. 27, n. 6, p. 625-641, 2001.

BARNEY, J. B. Firm resources and sustained competitive advantage. Journal of management, v. 17, n. 1, p. 99-120, 1991

BOAVENTURA, Jõao Maurício Gama; SIQUEIRA, João Paulo Lara. A estratégia e as redes de negócio. In: X SEMEAD, Anais... São Paulo, FEA, USP, 2008. Disponível em: < http://sistema.semead.com.br/10semead/sistema/resultado/trabalhosPDF/ 516.pdf $>$. Acesso em: 09 dezembro 2016.

BONATTO, Franciele; RESENDE, L.M.M; PONTES, Joseane; JUNIOR, P.P.A. A measurement model for managing performance of horizontal business networks and a research case. Prod. [online], vol.27, 2017.

CARNAUBA, Adriano Augusto Costa et. al. GOVERNANÇA DE REDES INTERORGANIZACIONAIS. FACEF Pesquisa-Desenvolvimento e Gestão, v. 15, n. 3, 2013.

CARNAUBA, A. A. C; PEREIRA, C. E. C. Mecanismos Formais e Relacionais de Governança em Redes de Negócios e sua Relação com a Confiança. In: XVII Semead 2014.

CARNEIRO, Jorge Manoel. Teixeira; CAVALCANTI, M. A. F. D; SILVA, J. F. da. Os determinantes da sustentabilidade da vantagem competitiva na visão resource-based. In: ENCONTRO ANUAL DA ANPAD (23: 1999: Foz do Iguaçu). Anais... Foz do Iguaçu: ANPAD, 1999.

CHILD, John; FAULKNER, David; TALLMAN, Stephen B. Cooperative strategy. Oxford: Oxford University Press, 2005.

CASSEL, C.H ackl, P., and Westlund, A.H. Robustness of partial least squares method for estimating latent variable quality structures. Journal of Applied Statistics, 435-446, 1999.

CELANI, C.K.P.B; ARANTES, F. P; LEITE, M. S. A. Confiança na gestão de cadeias de suprimentos: Uma revisão sistemática da literatura. XXXV Encontro Nacional de Engenharia de Produção. Fortaleza. Out/2015.

CLARO, D.P.; CLARO, P.B. Gerenciando Relacionamentos Colaborativos com Fornecedores. Revista de Administração de Empresas, v.44, n.4.2004.

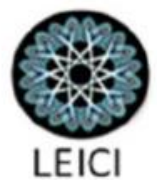


CUNHA, J. A. C.; CORRÊA, H. L. Avaliação de Desempenho Organizacional: um estudo aplicado em hospitais filantrópicos. Revista de Administração de Empresas, São Paulo, v. 53, n. 5, p. 485-499, set./out. 2013.

DA SILVA, D.P. N.; DE CAMPOS, S.; WILLERSON, L.; DE MELO, M. S. Governança Interorganizacional: Entendendo o debate teórico da literatura internacional. SIMPOI (2016) Anais... São Paulo, 2016.

DE BENEDICTO, Samuel Carvalho.; RODRIGUES, A. C.; ABBUD, Emerson Luiz. Governança corporativa: uma análise da sua aplicabilidade no setor público. Encontro Nacional de Engenharia de Produção, v. 28, 2008.

DE SOUZA AGUIAR, Helder et al. Análise da competitividade de clusters de negócios de varejo: ajuste de métricas através de uma aplicação no cluster varejista de moda do Bom Retiro. REGE-Revista de Gestão, v. 24, n. 2, p. 122-133, 2017.

DUTRA, I. Redes Empresariais de negócios orientadas por estratégia definida pela governança supraempresas: estudo e metodologia de avaliação dos atributos componentes e respectivas influências sobre o poder de competitividade. 2009. 278 f. Tese (Doutorado), Universidade de São Paulo, São Paulo.

EFRON, B. e Tibshirani, R. J. An Introduction to the Bootstrap. Chapman \& Hall, 1993.

FILHO, Abimael Magno do Ouro; OLAVE, Maria Elena Leon; BARRETO, Ikaro Daniel de Carvalho. Fatores Desarticuladores da Cooperação em Arranjos Produtivos Locais: Um Estudo Quantitativo no APL de Confecções de Tobias Barreto/SE. Brazilian Business Review. V.12, n.5, p.17-40, 2015.

FORNELL, C. e Larcker, D. F. Evaluating structural equation models with unobservable variables and measurement error. Journal of marketing research, p. 39-50, 1981.

FREITAS, R. L.; MARTINS, H.C.; DIAS, A.T. Comportamento Organizacional em Redes para Posicionamento Estratégico e Desempenho Superior: um Estudo no Setor de TI. XXXVIII ENCONTRO DA ANPAD, Rio de Janeiro, 2014.

GAMEIRO, Paulo Alexandre Dias. As organizações em rede. Universidade Lusófona de Humanidades e Tecnologias, 2008. Disponível em: < http://www.bocc.ubi.pt/pag/gameiropaulo-as-organizacoes-em-rede.pdf $>$. Acesso em: 17 de dezembro 2016.

GUIMARÃES, A.T.; SANTOS, S.A.; ZACCARELLI, S.B; KUNIYOSHI, M.S. Empresas instaladas em Clusters Orientações Estratégicas Duais influenciadas pelas Governanças Supraempresariais de Clusters e Redes de Negócios: Um Estudo do Setor de Vitivinicultura no Brasil e Portugal. In: ENCONTRO DA ENANPAD, 37 Rio de Janeiro, 2013.

GUIMARÃES, A. T. Empresas Instaladas em Clusters com Orientação Estratégica Dual, Originadas na Governança de Clusters de Redes de Negócios: Uma Busca Focada no Negócio do Vinho das Regiões do Porto, em Portugal e do Vale dos Vinhedos, No Brasil. FEA/USP. São Paulo: 2009 (Tese Doutorado).

GULATI, Ranjay; NOHRIA, Nitin; ZAHEER, Akbar. Strategic Networks. Strategic Management Journal, v. 21, n. 3, special issue: mar p. 203-215, 2000.

GUSSONI, W. M. S; WEISE, A. D; MEDEIROS, F. S. B. Cooperação e governança nos Arranjos Produtivos Locais: o caso das empresas de software no Estado do Paraná. Desenvolvimento em Questão, v. 13, n. 29, p. 125-157, 2015.

HAIR, J. F., Hult, G. T. M., Ringle, C., \& Sarstedt, M. A primer on partial least squares structural equation modeling (PLS-SEM). Sage Publications, 2014.
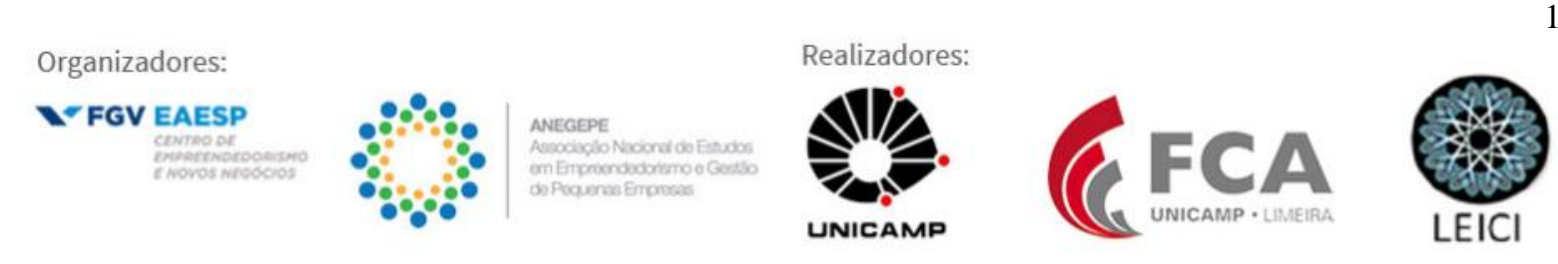
HALL, R. H. (2004) Organizações: Estruturas, processos e resultados. 8.ed. São Paulo: Prentice Hall.

HITT, Michael; IRELAND, R. Duane; HOSKISSON, Robert. Administração Estratégica. São Paulo: Pioneira Thomson Learning, 2003.

KLEIN, Leander Luiz; PEREIRA, Breno Augusto Diniz. Contribuições Para Gestão de Redes Interorganizacionais: Fatores Determinantes Para Saída de Empresas Parceiras. Revista Eletrônica de Administração. V.20, n 2, p. 305-340, 2014

MACIEL, Cristiano Oliveira; REINERT, Maurício; CAMARGO, Camila. Confiança e possibilidade de conflitos em redes estratégicas hierárquicas. RAM, Rev. Adm. Mackenzie (Online), São Paulo, v. 12, n. 4, p. 182-208, aug. 2011.

MARIANO, E. B.; GUERINI, F. M.; REBELLATO, D. A. D. N. Análise da relação entre estrutura e desempenho de redes interorganizacionais colaborativas. Gestão \& Produção, v. 19, n. 3, p. 471-479, 2012.

MARTINS, H. C., GONÇALVES, C. A., MIRANDA, G. N. V. Comportamento Organizacional em Redes para Sustentabilidade e Crescimento: Um Estudo no Setor de Saúde. XXXVI ENCONTRO DA ANPAD, Rio de Janeiro, 2012.

MARTINS, H.C.; SIQUEIRA, L. A.; GONÇALVES, C.A. Atuação em rede, Confiança e Recursos Internos como Antecedentes da Efetividade da Governança Supraempresarial: Um Estudo do Impacto no Desempenho das Empresas Envolvidas. XL ENCONTRO DA ANPAD, Bahia, 2016.

MCEVILY, B.; PERRONE, V.; ZAHEER, A. Trust as an organizing principle. Organization Science, v. 14, n. 1, p. 91-103, 2003.

NUNNALY, J. e Bernstein, I. H. Psychometric Theory. McGraw-Hill: New York. 1994.

PROVAN, Keith G.; KENIS, Patrick. Modes of Network Governance: Structure. Management, and Effectiveness [J. Journal of Public Administration Research and Theory, 2008, 18 (2): 229-252.

ROTH, Ana Lucia; WEGNER, Douglas; JUNIOR, José Antônio Valle Antunes; PADULA, Antônio Domingos. Diferenças e inter-relações dos conceitos de governança e gestão de redes horizontais de empresas: contribuições para o campo de estudos. Revista de Administração, v. 47, n. 1, p. 112-123, 2012.

ROSSETI, José Paschoal; ANDRADE, Adriana. Governança Corporativa: fundamentos, desenvolvimentos e tendências. São Paulo: Ed. Atlas, 2006.

SCHMIDT, Jens; KEIL, Thomas. What makes a resource valuable? Identifying the drivers of firm idiosyncratic resource value. Academy of Management Review, v. 38, n. 2, p. 206-228, 2013.

SEBRAE - Serviço Brasileiro de Apoio a Micro e Pequenas Empresas. Disponível em: <http://www.sebrae.com.br/sites/PortalSebrae/canais_adicionais/conheca_quemsomos> Acesso em: 11 de junho de 2017.

SILVA, R. S., GASPAR, M. A., FARINA, M. C. Ganhos de Competitividade em Redes de Cooperação: Estudo em uma Cooperativa de Pequenas Farmácias. XVIII SEMEAD, 2015.

SIQUEIRA, B. Lucas. Antecedentes da Efetividade da Governança Supraempresarial e sua Influência no Desempenho das Empresas Envolvidas. Faculdade de Ciências Empresarias FACE. Universidade FUMEC. Belo Horizonte/MG (2015).

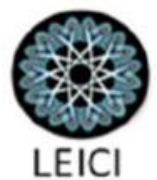


SOMBERGER, Geovane Paulo; HOPPEN, Noberto, RIGONI, Eduardo Henrique; KLEIN, Amarolinda Zanela; REDIVO, Arlete. Governança em comunidades virtuais de negócios. Revista de Gestão, FEA USP, v.24, p.149-158, 2017.

TELLES, R. Clusters e Redes de Negócios. Curitiba. IESDE Brasil S.A., 2008. 216p. TENENHAUS, M., Amato, S., \& Esposito Vinzi, V. A global goodness-of-fit index for PLS structural equation modelling. In Proceedings of the XLII SIS scientific meeting (Vol. 1, pp. 739-742), 2004.

VALMORBIDA, Sandra; BORTOLUZZI, Sandro; ENSSLIN, Sandra. Panorama das pesquisas e ferramentas de avaliação de desempenho organizacional: investigação em periódicos nacionais. In: XIV SIMPÓSIO DE ADMINISTRAÇÃO DA PRODUÇÃO, LOGÍSTICA E OPERAÇÕES INTERNACIONAIS (SIMPOI). Anais... São Paulo, 2011.

VALLANDRO, L. F. J; TREZ, G. Visão baseada em recursos, estratégia, estrutura e performance da firma: uma análise das lacunas e oportunidades de pesquisas existentes no campo da administração estratégica. Análise-Revista de Administração da PUCRS, v. 24, n. 1, p. 79-81, 2013.

VERDECHO, M. J; ALFARO-SAIZ, J. J; RODRÍGUEZ-RODRÍGUEZ, R; ORTIZ-BAS, A. The analytic network process for managing inter-enterprise collaboration: A case study in a collaborative enterprise network. Expert Systems with Applications, v.39, n.1, p.626-637, 2012.

VERSCHOORE, J; BALESTRIN, A. A associação em redes de cooperação influencia os resultados de pequenas e médias empresas? Ciências Sociais Unisinos, v. 46, n. 1, p. 105$115,2010$.

ZACCARELLI, S. B.; TELLES, R.; SIQUEIRA, J.P.L.; BOAVENTURA, J.M.G.; DONAIRE, D. Clusters e Redes de Negócios: uma nova visão para gestão dos negócios. São Paulo: Atlas, 2008.

ZAHEER, Akbar; MCEVILY, Bill; PERRONE, Vincenzo. Does trust matter? Exploring the effects of interorganizational and interpersonal trust on performance. Organization science, v. 9, n. 2, p. 141-159, Mar./Abr. 1998.

WEGNER, Douglas. Governança, gestão e capital social em redes horizontais de empresas: uma análise de suas relações com o desempenho das empresas participantes. 2011. Tese (Doutorado). Universidade Federal do Rio Grande do Sul.

WEGNER, D; KOETZ, C. I.; WIK, O. E. A influência da governança formal de redes interorganizacionais (RIOS) no desempenho das empresas participantes. XXXVI Encontro da ANPAD. Rio de Janeiro. Set/2012.

ZIMMER, Marco Vinício et al. Gestão de desempenho por competências: integrando a gestão por competências, o balanced scorecard e a avaliação 360 graus. Rap — rio de Janeiro, v. 42, n. 5, p. 875-98, 2008. 